\title{
FÖRDERBEDINGUNGEN FÜR PUBLIKATIONSFONDS: OPEN ACCESS - GANZ ODER GAR NICHT?
}

\section{von Christof Capellaro und Christian Kaier}

Zusammenfassung: Welche Förderbedingungen für Open-Access-Publikationsfonds sind sinnvoll oder gar notwendig? Sind die damit verbundenen Hürden und Einschränkungen mit dem Ziel einer vollständigen Transformation des wissenschaftlichen Publikationssystems zu Open Access vereinbar? Der Beitrag behandelt einige Fragestellungen, die im Zuge der Ausarbeitung eines Papiers zu Förderbedingungen von Publikationsfonds im Projekt „Austrian Transition to Open Access - AT2OA” diskutiert wurden. Behandelt werden Spannungsfelder, die aus disziplinspezifischen Unterschieden resultieren, die Frage der Bevorzugung bestimmter Personengruppen in den Förderbedingungen sowie Vorgaben für Lizenzen und Publikationstypen. Abschließend geht der Beitrag auf die Frage ein, welche Arten von Ausgaben und welche Open-Access-Finanzierungsmodelle für eine Förderung aus den Mitteln eines Publikationsfonds generell in Betracht kommen.

Schlagwörter: Hochschulraumstrukturmittelprojekt; Austrian Transition to Open Access (AT2OA); Publikationsfonds; Förderbedingungen; Open Access; Transformation; Open-Access-Finanzierung; Article Processing Charges

\section{FUNDING CONDITIONS FOR OPEN ACCESS PUBLICATION FUNDS: OPEN ACCESS - “ALL OR NOTHING"?}

Abstract: Which funding conditions for Open Access publication funds are advisable or even necessary? Are such restrictions compatible with the goal of a complete transformation of the scholarly publishing system to Open Access? This article deals with a number of questions that were discussed in the course of the drafting of a guidance document on funding conditions for publication funds in the project "Austrian Transition to Open Access - AT2OA". It deals with controversial issues that originate from discipline-specific differences, the preference or discrimination of persons (or groups) for strategic reasons, as well as requirements concerning licensing and publication types. Finally, it discusses which types of expenditure or Open Access funding models should be eligible for funding from a publication fund.

Keywords: Higher Education Structural Funds Project; Austrian Transition to Open Access (AT2OA); Publication Fund; Funding Conditions; Open Access; Transformation; Open Access Funding; Article Processing Charges 
DOI: https://doi.org/10.31263/voebm.v72i1.2278

(c) Christof Capellaro, Christian Kaier

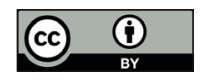

Dieses Werk ist lizenziert unter einer

Creative-Commons-Lizenz Namensnennung 4.0 International

\section{Inhalt}

1. Einleitung

2. Spannungsfelder, die aus disziplinspezifischen Unterschieden resultieren

3. Bevorzugung bestimmter Personengruppen bei der Publikationsförderung? Das Beispiel Early Career Researchers (ECR)

4. Vorgaben für Lizenzen im Spannungsfeld von Nachnutzbarkeit und unerwünschter Nutzung

5. Welche Ausgaben sollen überhaupt aus dem Publikationsfonds bezahlt werden?

6. Zusammenfassung

\section{Einleitung}

Im Rahmen des Projektes "Austrian Transition to Open Access - AT2OA” (www.at2oa.at) beschäftigt sich das Teilprojekt 3 mit der Finanzierung von Open-Access-Publikationskosten durch die Etablierung institutioneller Publikationsfonds. ${ }^{1}$ Die Erstellung einer Handreichung zu Förderbedingungen von Open-Access-Publikationsfonds ${ }^{2}$ war ein wesentlicher Teil der Arbeit in diesem Teilprojekt und konnte im Frühjahr 2019 erfolgreich abgeschlossen werden. Das Papier, das von insgesamt 13 Mitgliedern des Teilprojekts 3 gemeinsam erarbeitet wurde, ${ }^{3}$ stellt in der Art eines Baukastens Elemente zur Verfügung, die bei der Etablierung eines Open-Access-Publikationsfonds diskutiert und geregelt werden sollten. Zugleich möchte es Anregungen zur Evaluierung der Förderbedingungen bestehender Publikationsfonds geben.

Bei der Priorisierung dessen, was als „förderwürdig” gilt, können die unterschiedlichen Interessenslagen und strategischen Schwerpunktsetzungen unterschiedlicher Einrichtungen deutlich hervortreten. Die Vielfalt denkbarer und überlegenswerter Förderbedingungen soll jedoch nicht davon ablenken, dass eine möglichst große Einheitlichkeit und nationale wie internationale Abstimmung auf diesem Gebiet von großer Wichtigkeit ist, um einfache Rahmenbedingungen für Wissenschaftlerlnnen und Verlage zu gewährleisten, Kosteneffizienz zu erreichen und gemeinsame Interessen gegenüber Verlagen besser vertreten zu können. ${ }^{4}$ 
Förderbedingungen für Publikationsfonds ${ }^{5}$ zielen darauf $a b$, Mindeststandards für den Einsatz der (begrenzten) öffentlichen bzw. institutionellen Mittel zur Finanzierung von Publikationen zu gewährleisten. Allerdings wird zwangsläufig jener Anteil der Publikationen, der den Bedingungen nicht entspricht oder aus budgetären Gründen nicht finanziert werden kann, von einer Förderung ausgeschlossen. Dadurch entsteht ein Spannungsverhältnis zu dem Ziel, Open Access für 100 Prozent der wissenschaftlichen Publikationen zu erreichen. ${ }^{6}$

Eines der Ziele der Open-Access-Bewegung war und ist es, wissenschaftliches Publizieren kostengünstiger und effizienter zu machen. Dazu muss eine vollständige Transformation vom Kauf und der Lizenzierung von Verlagsprodukten zu Open Access, also der Finanzierung von Publikationskosten, stattfinden, denn das teuerste System ist nach derzeitiger Ansicht ein (dauerhaftes) Nebeneinander von „reader pays” und „author pays”. Paradoxerweise würde aber eine solche Transformation "um jeden Preis”" - also ohne Einschränkungen wie etwa der Deckelung von APCs - dazu führen, dass von Seiten der Verlage Publikationsgebühren in beliebiger Höhe durchgesetzt werden könnten. Welcher finanzielle Wert soll also Publikationsdienstleistungen bzw. dem freien Zugang für alle zugeschrieben werden? Soll oder muss Open Access „ganz oder gar nicht” oder doch in Form einer Kompromissvariante implementiert werden, die vermutlich am wenigsten effizient wäre?

Im Zuge der Arbeit an dem eingangs genannten Papier kam es zu einem intensiven und sehr lebendigen Austausch darüber, welche Anreize und Einschränkungen im Zusammenhang mit den Förderbedingungen eines Publikationsfonds notwendig sind. Ausgehend vom Diskussionsprozess der Arbeitsgruppe sollen im Folgenden einige dieser Überlegungen dargestellt werden. Der Beitrag konzentriert sich dabei bewusst auf Spannungsfelder, die sich bei der Ausgestaltung möglichst einheitlicher Förderbedingungen aus disziplinspezifischen Unterschieden und unterschiedlichen Publikationsstrategien verschiedener Einrichtungen und Wissenschaftlerlnnen ergeben.

\section{Spannungsfelder, die aus disziplinspezifischen Unterschieden resultieren}

Durch die personelle Zusammensetzung der Arbeitsgruppe brachten KollegInnen aus sehr unterschiedlichen Einrichtungen (Kunst-, Medizin-, Technische- und Volluniversitäten, Fördergeber) ihre Perspektive auf das Thema Open Access im Allgemeinen und den Zweck von Publikationsfonds im Besonderen ein. Dadurch wurden nicht nur die - teils recht un- 
terschiedlichen - Interessenslagen der einzelnen Einrichtungen deutlich, sondern auch die vielfältigen Erwartungshaltungen, die sich mit dem Thema Open Access verbinden. Die angesprochene Personenkonstellation ermöglichte es auch, dass unterschiedliche Sichtweisen in das gemeinsam erarbeitete Papier integriert werden konnten. In diesem Zusammenhang wurden freilich auch erhebliche disziplinäre Unterschiede sichtbar.

Open Access hat sich bislang als Publikationsmodell in verschiedenen Disziplinen in unterschiedlichem Ausmaß etabliert. Die Hindernisse und Herausforderungen, die sich einer umfassenden Transformation entgegenstellen, variieren aufgrund heterogener Publikationskulturen beträchtlich:

- Naturwissenschaften, Technik, Medizin (STM): In diesen Bereichen spielen umstrittene Metriken auf Zeitschriftenebene - wie etwa der Impact Factor - eine große Rolle. Mitunter werden Publikationen explizit von einer Open-Access-Förderung ausgeschlossen, wenn das Publikationsmedium, in dem veröffentlicht werden soll, zu niedrige bibliometrische Indikatoren aufweist.

- Geistes-, Sozial- und Kulturwissenschaften (GSK): Die besonderen Publikationsformate und -frequenzen sowie die höhere Bedeutung von Sammelbänden und Monografien in den GSK führen zu anderen Kostenstrukturen als in Disziplinen, die vorwiegend kurze Artikel publizieren. Andere, informelle Formen der Qualitätssicherung als das vor allem in den STM-Fächern übliche Peer Review (wie z.B. Editorial Review) werden außerhalb des GSK-Bereichs oft nicht anerkannt. Aufgrund dieser Publikationskultur und den hohen Kosten für Open-Access-Bücher werden derzeit in den GSK relativ wenige Open-Access-Veröffentlichungen gefördert. Aus den genannten Gründen wird man insgesamt von einer strukturellen Benachteiligung der GSK-Fächer beim Open-Access-Publizieren sprechen müssen. Hinzu kommt, dass vor allem im Bereich der Künste und Kunstwissenschaften aufgrund der häufigen Verwendung von Abbildungen komplexe Rechtsfragen auftreten. Deren Klärung wie auch die Finanzierung von Kosten für Bildrechte erschweren das Open-Access-Publizieren in diesen Disziplinen enorm. Besonders im Grenzbereich zwischen Kunstwissenschaft und Kunstproduktion kommen komplexe Fragen allenfalls zu schützender Verwertungsrechte hinzu. Auch hier stellt sich die Frage, wie Förderbedingungen fachlich adäquat angepasst werden können.

Förderbedingungen bieten außerdem die Möglichkeit, in Fällen Unterstützung zu bieten, wo sich die Transition besonders schwierig gestaltet. So 
könnte eine Institution etwa beschließen, dass Fördermittel schwerpunktmäßig dort als Anreiz eingesetzt werden, wo sich das Open-Access-Publizieren bisher vergleichsweise wenig etablieren konnte.

Auch die Höhe der Open-Access-Publikationskosten kann je nach Disziplin sehr unterschiedlich ausfallen, sodass die Überlegung naheliegt, eine allfällige Deckelung ${ }^{8}$ des Förderbetrags disziplinabhängig zu gestalten. Auch dieses Thema wurde in der Arbeitsgruppe kontrovers diskutiert: Was sind überhaupt „angemessene” Publikationskosten? Orientiert sich die Höhe der Publikationskosten tatsächlich an den Verlagsleistungen inklusive fachspezifischer Formen der Qualitätssicherung wie Peer Review und hoher rejection rates - oder an anderen Faktoren wie der Zahlungsbereitschaft der Autorlnnen und der Verwertbarkeit der Ergebnisse? Wie ist damit umzugehen? Letztlich suggerieren unterschiedlich hohe Förderobergrenzen nach Disziplin auch eine unterschiedliche „Wertigkeit" von Fächern und Forschungsergebnissen. Bei interdisziplinären Arbeiten stellt sich zudem die Frage, welche der möglicherweise disziplinär unterschiedlichen Obergrenzen zur Anwendung kommt. Andererseits wird ein „one-size-fits-all”-Ansatz dem Status quo ebenfalls nicht gerecht: Da die durchschnittliche Publikationsgebühr nach Fach variieren kann, wird der Kreis der förderfähigen Publikationen in unterschiedlichen Disziplinen durch eine einheitliche Obergrenze unterschiedlich stark eingeschränkt. Festzuhalten bleibt, dass eine Kostenobergrenze (ganz gleich, ob sie nun disziplinspezifisch ausfällt oder nicht) kein inhaltliches Kriterium darstellt und jedenfalls Fragen nach einrichtungsinterner Verteilungsgerechtigkeit aufwirft.

\section{Bevorzugung bestimmter Personengruppen bei der Publikationsför- derung? - Das Beispiel Early Career Researchers (ECR)}

Zielen die Förderbedingungen eines Fonds aus finanziellen oder strategischen Gründen auf die Bevorzugung einzelner Personengruppen ab, so steht auch dies dem übergeordneten Ziel einer „Full Transformation“ mitunter entgegen. ${ }^{9}$ Andererseits ist es für eine langfristige Transformationsstrategie sinnvoll, Anreize zu setzen, die die Finanzierbarkeit des Publikationssystems sichern. Ein möglicher Ansatz ist dabei die Bevorzugung von Early Career Researchers (ECR).

Die Förderung des wissenschaftlichen Nachwuchses ist im österreichischen Universitätsgesetz als zentrale Aufgabe der Universitäten definiert ${ }^{10}$ und eine solche kann unter anderem im Rahmen der Bestimmungen eines 
Publikationsfonds umgesetzt werden. ECR sind für ihr berufliches Fortkommen oftmals auf Publikationen in „prestigeträchtigen” Zeitschriften und Verlagen angewiesen, verfügen aber selbst meist noch nicht über eine entsprechende Reputation und sind daher im Bereich des wissenschaftlichen Publizierens gegenüber älteren Wissenschaftlerlnnen strukturell benachteiligt. Außerdem scheint es sinnvoll, im Hinblick auf eine dauerhafte Umstellung auf Open Access gerade jüngere Wissenschaftlerlnnen für dieses Thema zu sensibilisieren.

Eine besondere Unterstützung für ECR durch die bevorzugte Zuerkennung von Mitteln aus der Open Access-Publikationsförderung scheint somit grundsätzlich sinnvoll. Auf diese Weise könnten Anreize zum Open-Access-Publizieren insbesondere für ECR geschaffen werden - in Kombination mit einer besonderen Berücksichtigung und Wertschätzung gegenüber Open Access publizierten Forschungsleistungen bei der Stellenvergabe, wie sie in vielen institutionellen Open-Access-Policies ohnehin bereits angeführt ist. ${ }^{11}$

Herausforderungen bei der Umsetzung ergeben sich freilich daraus, dass die Bevorzugung einer Personengruppe zugleich die Benachteiligung einer anderen bedeutet und als solche ausreichend begründet werden muss: Welche sachlichen Gründe rechtfertigen eine Ungleichbehandlung innerhalb des wissenschaftlichen Personals? Analog dazu ist etwa auch eine allfällige Begrenzung der Anzahl förderfähiger Publikationen pro Wissenschaftlerln in den Förderrichtlinien zu beurteilen.

Ein weiteres Problem ergibt sich aus dem Umstand, dass die Gruppe der ECR keineswegs eindeutig definiert ist: Kriterien für ECR oder „Nachwuchswissenschaftlerlnnen" verwenden mitunter das Lebensalter, aber auch Karrierestufen und die Dauer der bisherigen wissenschaftlichen Tätigkeit als Maßstab und unterscheiden sich dabei deutlich voneinander. Eine klare Abgrenzung von ECRs zu anderen Personengruppen würde allerdings eine eindeutige Definition der entsprechenden Begriffe voraussetzen. Gerade in Bezug auf ECR kann auch strittig sein, ob Forschende ohne aufrechtes Dienstverhältnis (typischerweise etwa DoktorandInnen) für eine Förderung überhaupt in Betracht kommen. ${ }^{12}$

\section{Vorgaben für Lizenzen im Spannungsfeld von Nachnutzbarkeit und unerwünschter Nutzung}

Wurde in den Anfangstagen der Open-Access-Bewegung oftmals die Hoffnung geäußert, dass es durch den Umstieg auf Open-Access-Modelle zu 
einer Kostensenkung im System des wissenschaftlichen Publizierens insgesamt kommen könne, so zeichnet sich eine derartige Entwicklung aus heutiger Sicht nicht ab. ${ }^{13}$ Der Kostenfaktor kann folglich - zumindest derzeit - nicht als entscheidendes Argument für den Umstieg auf Open Access angeführt werden.

Unter dem Gesichtspunkt der Nachnutzbarkeit ist das Open-AccessModell jenem des Closed Access allerdings in der Tat deutlich überlegen: Durch die Vergabe entsprechender Lizenzen kann die Wiederverwendbarkeit der publizierten Inhalte auf einfache Weise ermöglicht werden. ${ }^{14}$

Eine wissenschaftliche Abhandlung kann - unter Angabe des Autors oder der Autorin - in ein Lehrveranstaltungsskript, auf die Webseite einer Ausstellung oder in eine Buchpublikation übernommen werden, ohne dass aufwändige Nachfragen, Rechteklärungen oder Vertragsschlüsse erforderlich sind - vorausgesetzt, der Text wurde Open Access publiziert, mit einer entsprechenden Lizenz versehen und enthält keine rechtlich geschützten Materialien (wie z.B. Abbildungen) Dritter.

Durch die Vergabe entsprechender Open-Content-Lizenzen wird außerdem die exklusive Verwertung wissenschaftlicher Inhalte durch Verlage und andere Dienstleister weitgehend ausgeschlossen. Gerade dieser Aspekt dürfte angesichts der aktuellen Entwicklungen im Bereich der Künstlichen Intelligenz sowie des Text- und Data-Minings an Brisanz und Bedeutung gewinnen. ${ }^{15}$ Mit Hilfe von Creative-Commons-Lizenzen (CC-Lizenzen) lässt sich der Status eines Dokuments als offen und nachnutzbar durch den Autor oder die Autorin selbst auf einfache Weise festlegen und für andere Nutzerlnnen dokumentieren.

Hierbei erweist sich gerade die leichte Verständlichkeit und einfache Handhabbarkeit der CC-Lizenzen nach dem Baukastenprinzip ${ }^{16}$ als entscheidender Vorteil. CC-Lizenzen sind auch für juristische Laien leicht anzuwenden und machen das Aufsetzen eines individuellen Lizenzvertrags sowie eine umfangreiche Rechtsberatung (weitgehend) überflüssig. So kann gewährleistet werden, dass auch hohe Fallzahlen an Publikationen rasch und unkompliziert „rechtemäßig“ abzuwickeln sind.

Allerdings ist die leichte Anwendbarkeit notwendigerweise um den Preis einer erheblichen Komplexitätsreduktion erkauft und die „Einfachheit“ der Lizenz auch ihr größter Nachteil. Gerade die Frage nach der Verankerung der Lizenz Creative-Commons-Namensnennung (CC-BY) als obligatorisch in den Förderbedingungen von Publikationsfonds hat folgerichtig auch innerhalb der Arbeitsgruppe zu heftigen Diskussionen geführt.

CC-Lizenzen können, um das Motto des Beitrags aufzugreifen, nur „ganz oder gar nicht" vergeben werden und sind aufgrund ihres modulartigen Auf- 
baus zwangsläufig weniger flexibel als ein individueller Lizenzvertrag. Zwar lassen sich durch die Wahl der „richtigen“ Lizenz bestimmte Nutzungsarten ausschließen, nicht aber einzelne unerwünschte Nutzungskontexte. Im ungünstigsten Fall kann sich die Nachnutzung des eigenen Werks in einem nicht erwünschten Kontext jedoch nachteilig auf die Reputation und Karrierechancen von Wissenschaftlerlnnen auswirken und damit bei diesen u.U. auch zu einer negativen Wahrnehmung von Open Access führen.

Der unter die CC-BY-Lizenz gestellte Aufsatz eines Zeithistorikers oder einer Zeithistorikerin kann sich eben nicht nur im Lehrveranstaltungsskript eines Kollegen wiederfinden, was durchaus erwünscht sein dürfte, sondern auch als Beitrag in einem Sammelband, den ein rechtsextremer Verlag herausgibt.

Weniger anstößig für viele Autorlnnen, aber wahrscheinlich ebenfalls ärgerlich und bei Vergabe der Lizenz CC-BY jedenfalls zulässig, ist die Verbreitung von Textabschnitten oder ganzen Werken durch kommerzielle Verlage, die sich darauf spezialisiert haben, Open-Access-Inhalte gedruckt anzubieten - oftmals in zweifelhafter Zusammenstellung, auf schlechtem Papier und zu überhöhten Preisen.

Das Recht zu Bearbeitung und Remix (bei den Lizenzen CC-BY und CCBY-NC) erlaubt Eingriffe Dritter in den Text. Gerade in geistes- und kulturwissenschaftlichen Fächern, in denen oftmals auch der Textgestalt und der sprachlichen Ausformung bestimmter Inhalte ein erheblicher Eigenwert zugemessen wird, kann ein solcher Kontrollverlust als problematisch gesehen werden. Kultur- und GeisteswissenschaftlerInnen äußern daher mitunter den Wunsch unter Anwendung der Lizenz CC-BY-ND zu publizieren, um gegen unerwünschte Modifikationen hinsichtlich der Textgestaltung abgesichert zu sein.

Diese Beispiele zeigen, dass es letztlich stark vom betroffenen Wissenschaftsfach und der dort gepflegten Publikationskultur abhängt, inwieweit (bestimmte) CC-Lizenzen von Autorlnnen als problematisch angesehen werden. Entsprechende Einwände und Bedenken sollten ernst genommen werden - stehen dem Ziel der Open-Access-Bewegung nach maximaler Offenheit und bestmöglicher Nachnutzbarkeit aber mitunter entgegen.

Letztlich wird hier ein weiteres nur schwer aufzulösendes Spannungsfeld deutlich, das auch die Debatte um Open Access insgesamt kennzeichnet und aus den unterschiedlichen Interessenslagen verschiedener Stakeholder resultiert.

Betrachtet man das System wissenschaftlicher Kommunikation insgesamt aus einer Makroperspektive - wie etwa Wissenschaftspolitik und Forschungsförderer dies tun -, erscheint die Forderung nach größtmöglicher 
Nachnutzbarkeit wissenschaftlicher Inhalte als zentral, eine mögliche unerwünschte Nachnutzung, die sich damit verbindet, als vernachlässigbar. Nimmt man dagegen die Mikroperspektive des einzelnen Wissenschaftlers/ der einzelnen Wissenschaftlerin ein, wird - unter dem Gesichtspunkt der persönlichen Karriereplanung und Reputation - die Gewichtung mitunter umgekehrt ausfallen. Ein pragmatischer Ansatz, mit diesem Interessenskonflikt umzugehen, kann evtl. darin bestehen, in den Förderbedingungen eines Publikationsfonds die Verwendung der Lizenz CC-BY zwar ausdrücklich zu empfehlen, in begründeten Ausnahmefällen aber auch restriktivere Lizenzen zuzulassen.

\section{Welche Ausgaben sollen überhaupt aus dem Publikationsfonds be- zahlt werden?}

Schon im Vorfeld der Einrichtung eines Publikationsfonds muss festgelegt werden, wofür dessen Gelder abgerufen werden können. Hierbei ist insbesondere zu klären, welche Publikationstypen für eine Förderung in Betracht kommen, welche Open-Access-Modelle durch den Fonds unterstützt werden können und ob der Anspruch verfolgt wird, möglichst alle Aufwendungen, die an der betreffenden Einrichtung für Open-Access-Publikationen anfallen, aus dem Publikationsfonds zu begleichen. Je nachdem wie die Beantwortung dieser Fragen ausfällt, wird man zu einer eher engen oder weiter gefassten Definition des Begriffs „Publikationsfonds“ kommen.

Wird ein Publikationsfonds neu eingerichtet, so kann es durchaus sinnvoll sein, die Förderung zunächst auf einen einzelnen, einfach zu handhabenden Publikationstyp (etwa Zeitschriftenartikel) zu beschränken, da so der Einstieg in die Administration des Fonds deutlich erleichtert wird. Mittelfristig stellt sich aber auch hier die Frage nach dem „ganz oder gar nicht": Soll konsequent das Ziel einer flächendeckenden Transformation hin zu Open Access verfolgt werden, ist es jedenfalls folgerichtig, wenn sämtliche Publikationstypen, die an einer Institution im Regelfall vorkommen können, auch als förderfähig angesehen werden. ${ }^{17}$ Dementsprechend sollten etwa an einer Einrichtung, an der kultur- und geisteswissenschaftliche Fächer vertreten sind, Open-Access-Monografien nicht dauerhaft von einer Förderung ausgeschlossen bleiben, auch wenn dadurch eine höhere Dotierung des Fonds notwendig wird und der Arbeitsaufwand durch den deutlich komplexeren Publikationstyp Buch steigt.

Eng mit der Frage nach den förderfähigen Publikationstypen verknüpft ist auch die Frage, welche Open-Access-Modelle für eine Finanzierung aus 
dem Fonds in Betracht kommen. Sollen nur klassische Einzel-APCs und BPCs abgerechnet werden oder auch Deposits, Mitgliedschaften, Beiträge für alternative Open-Access-Infrastrukturen und Publikationskosten, die im Rahmen von Open-Access-Deals (wie z.B. SpringerCompact) anfallen? Gerade im Bereich nicht-kommerzieller Open-Access-Infrastrukturen nimmt die Zahl der Angebote derzeit deutlich zu, was mitunter mit einer nur noch schwer zu überblickenden Vielzahl von Geschäftsmodellen einhergeht. ${ }^{18}$

Vielerorts ist derzeit zu beobachten, dass Open-Access-Kosten unsystematisch aus unterschiedlichsten Kostenstellen und Etats bezahlt werden. Neben Open-Access-Publikationsfonds kommen hier etwa Projektmittel, Sonderetats der Rektorate und Vizerektorate, das Literaturbudget und/ oder Sachmittelbudget der betreffenden Universitätsbibliothek, Mittel des zentralen Informatikdiensts bzw. IT-Service sowie solche der Fachbereiche oder Institute in Betracht. Teils werden Kosten für Open-Access-Publikationen auch dann aus Mitteln von Fachbereichen oder Instituten bezahlt, wenn an der Einrichtung ein zentraler Publikationsfonds vorhanden ist.

Ein Gesamtüberblick auf Institutionsebene (z.B. Universitätsebene) darüber, wie hoch die Ausgaben für das Open-Access-Publizieren insgesamt sind, wird dadurch erheblich erschwert oder (bei sehr uneinheitlicher Ausgestaltung der Buchungspraxis) sogar verunmöglicht. ${ }^{19}$ Gleichzeitig ist davon auszugehen, dass die rasche und unkomplizierte Bereitstellung entsprechenden Zahlenmaterials künftig im Rahmen des internen Controllings wie auch der Berichterstattung nach außen eine immer wichtigere Rolle spielen wird.

Eine mögliche Lösung könnte darin bestehen, den Publikationsfonds als One-Stop-Shop für die Bezahlung sämtlicher Open-Access-relevanter Publikationskosten einer Einrichtung zu nutzen. Allerdings ist auch dieser Ansatz - wie sich in den Diskussionen der Arbeitsgruppe gezeigt hat - durchaus mit Schwierigkeiten behaftet. Zunächst stellt sich hier die Frage nach einer sinnvollen Abgrenzung zwischen Open-Access-Kosten im Allgemeinen und Publikationskosten im Speziellen. Hier ist eine sinnvolle Grenzziehung mitunter schwierig: Zweifellos wird man Kosten, die für den technischen Betrieb und die organisatorische Betreuung eines institutionellen Repositoriums anfallen, als Open-Access-Kosten einstufen können. Aber handelt es sich hierbei auch um Publikationskosten im engeren Sinn? Sind hier nur Sachkosten oder auch Personalkosten zu berücksichtigen? Soll (wie es das Beispiel des Repositoriums sinnvoll erscheinen lässt) zwischen Open-Access-Publikationskosten und Kosten für Open-Access-Infrastruktur unterschieden werden? Und falls man eine solche Unterscheidung vor- 
nimmt: Wie sind beispielsweise freiwillige Mitgliedsbeiträge für das „Directory of Open Access Journals" einzuordnen und wo sind sie zu buchen? Handelt es sich hier ebenfalls um Kosten für Open-Access-Infrastruktur? Sind diese Kosten aus dem Publikationsfonds zu buchen oder gehören sie eher in einen übergreifenden Etat für Mitgliedsbeiträge aller Art - mit der Gefahr, dass sie dort untergehen und nachträglich nicht mehr ohne weiteres als Kosten identifiziert werden können, die im Zusammenhang mit Open Access bzw. dem Open-Access-Publizieren stehen?

Oftmals dürften finanzielle und organisatorische Hindernisse der Nutzung des Publikationsfonds zur Zahlung sämtlicher Open-Access-Publikationskosten einer Einrichtung entgegenstehen. Publikationsfonds sind bisher i.d.R. nicht so gut ausgestattet, dass alle relevanten Zahlungen ohne weiteres aus ihnen bestritten werden könnten. Verfolgt man das Ziel, Gelder, die derzeit von den Fachbereichen oder Instituten selbst für einzelne Open-Access-Publikationen aufgewendet werden, im Publikationsfonds zu zentralisieren, so ist wahrscheinlich mit erheblichem Widerstand zu rechnen. Letztlich hängt es häufig von finanziellen Zufällen und dem Goodwill der Beteiligten ab, wo bestimmte Open-Access-Leistungen „untergebracht" werden können und dann auch zu buchen sind.

Da das Ziel, alle einschlägigen Zahlungsflüsse über den Publikationsfonds abzuwickeln, aus den genannten Gründen wenig realistisch sein dürfte, müssen auch andere Lösungswege in Betracht gezogen werden, um einen Überblick über die Gesamtkosten zu gewinnen. Ein lohnender Ansatz könnte hier darin liegen, Open-Access-(Publikations-)Kosten zwar weiterhin unterschiedlichen Etats oder Budgetstellen zuzuordnen, diese aber durch Vergabe einer einheitlichen Kostenart im Buchhaltungssystem sowie durch möglichst einheitliche Buchungstexte gemeinsam abfragbar zu machen.

\section{Zusammenfassung}

Zusammenfassend lässt sich sagen, dass übergreifende Förderbedingungen, die in gleicher Weise für die Publikationsfonds möglichst vieler Einrichtungen gelten, überaus wünschenswert sind. Bemühungen um eine entsprechende Vereinheitlichung finden jedoch in disziplinspezifischen Unterschieden, heterogenen Publikationskulturen, uneinheitlichen Interessenslagen und Publikationsstrategien verschiedener Einrichtungen ihre Grenzen.

Über alle Unterschiede hinweg stellt sich allerdings immer die Frage, inwieweit die Förderbedingungen eines Fonds so eng gefasst sind, dass nur 
mehr ein marginaler Teil aller Publikationen einer Einrichtung überhaupt für eine Förderung in Betracht kommt. Ist nur ein kleiner und (z.B. inhaltlich oder über den Impact Factor des Publikationsmediums) besonders herausgehobener Teil aller Veröffentlichungen förderfähig, so steht dies letztlich dem Ziel einer umfassenden Transformation hin zu Open Access entgegen.

Freilich werden Einschränkungen in den Förderbedingungen derzeit oftmals weniger aus inhaltlichen Gründen vorgenommen, sondern vielmehr, um dem Sachzwang begrenzter Mittel Rechnung zu tragen. Stünden insgesamt mehr Mittel für das Open-Access-Publizieren zur Verfügung, wäre manche Debatte über den Sinn oder Unsinn einer einzelnen Vorgabe, wie sie auch im Rahmen der Arbeitsgruppe immer wieder geführt wurde, wahrscheinlich obsolet. Auch die Tatsache, dass Open-Access-Publikationskosten vielerorts aus unterschiedlichsten Kostenstellen bezahlt werden, dürfte dem Umstand geschuldet sein, dass insgesamt zu wenig Mittel zur Verfügung stehen, die ausschließlich und explizit für das Open-Access-Publizieren gedacht sind.

Besteht bei der Finanzierung von Open Access mithin noch deutlich "Luft nach oben“, lässt sich doch auch festhalten, dass mit dem Projekt AT2OA hier ein wichtiger Schritt in die richtige Richtung getan worden ist.

Christof Capellaro, M.A. ORCID: http://orcid.org/0000-0001-8812-1613 Universität Salzburg, Universitätsbibliothek E-Mail: christof.capellaro@sbg.ac.at

Mag. Christian Kaier ORCID: http://orcid.org/0000-0002-8750-6666 Universität Graz, Universitätsbibliothek E-Mail: christian.kaier@uni-graz.at 
1 „Ein Open-Access-Publikationsfonds ist ein Finanzierungs- und Steuerungsinstrument wissenschaftlicher Einrichtungen zur Übernahme von Open-Access-Publikationsgebühren." Der Begriff APC - Article Processing Charges - wird dabei oft synonym zu Open-Access-Publikationsgebühren verwendet (Pampel/Tullney, 2017, S. 162).

2 Capellaro, Kaier et al. (2019).

3 Wir bedanken uns herzlich bei allen KollegInnen, die an der Erstellung des Papiers mitgearbeitet haben: Magdalena Andrae (Technische Universität Wien), Guido Blechl (Universität Wien), Vitali Bodnar (mdw - Universität für Musik und darstellende Kunst Wien), Andreas Ferus (Akademie der bildenden Künste Wien), Daniel Formanek (Medizinische Universität Wien), Michael Kranewitter (Universität Linz), Edith Leitner (Universität Mozarteum Salzburg), Martina Liska (Universität für Bodenkultur Wien), Eva Mayrgündter (Medizinische Universität Innsbruck), Katharina Rieck (FWF), Gregor Steinrisser-Allex (Medizinische Universität Graz).

4 Bruch et al. (2014), S. 15. Siehe auch die TU9-Handreichung zu einheitlichen Kriterien für Open-Access-Publikationsfonds, in der die Anwendung der existierenden DFG-Förderbedingungen auch für nicht-DFG-geförderte Publikationsfonds befürwortet wird, vgl. TU9 German Universities of Technology e. V. (2018).

5 Siehe auch Bruch et al. (2014), insbesondere S. 24-26.

6 Allerdings werden auch im Subskriptionssystem nicht „alle” Inhalte von allen lizenziert (also: bezahlt) und es stehen nicht 100 Prozent der wissenschaftlichen Literatur allen Forschenden an jeder Institution zur Verfügung.

7 „Es ist nicht ausreichend, über Publikationsfonds die Effizienz von Zahlungsabwicklungen zu steigern, wenn nicht gleichzeitig Ziele wie z. B. bestimmte Lizenzen, technische Standards oder Kosteneffizienz durchgesetzt werden können." Bruch et al. (2014), S. 16.

8 Wurde die Sinnhaftigkeit von Förderobergrenzen anfangs unterschiedlich beurteilt (vgl. Bruch et al, 2014, S. 14), sprechen sich die Allianz der Deutschen Wissenschaftsorganisationen und die TU9 inzwischen dafür aus (vgl. Bruch et al, 2015; TU9 German Universities of Technology e. V. 2018).

9 Vgl. Bruch et al (2014), S. 21-22.

$10 \S 3$ Z 4 Universitätsgesetz 2002.

11 Ein Überblick zu Open-Access-Policies und -Förderungen in Österreich findet sich bspw. auf der Webseite des Open Science Network Austria (OANA): https://oana.at/ueber-open-science/open-access-ressourcen/. 
12 Siehe zum Kreis der anspruchsberechtigten Personen auch Capellaro, Kaier et al. (2019), Punkt 2.1.

$13 \mathrm{Zu}$ Kostenverschiebungen zwischen einzelnen Einrichtungen, auf Länderebene etc. kann es durch einen wachsenden Open-Access-Anteil natürlich sehr wohl kommen. Für Österreich vgl. dazu die ebenfalls im Rahmen des Projekts AT2OA erarbeitete Studie von Fessler/Hölbling (2019).

14 Vgl. dazu die Empfehlungen von Bruch et al. (2015), S. 6.

15 Beispielsweise hat der Verlag Springer jüngst das erste auf Basis von Künstlicher Intelligenz geschriebene Buch veröffentlicht. https://www. computerbase.de/2019-04/kuenstliche-intelligenz-erstes-buch-algorithmus/

16 Siehe https://creativecommons.org/choose/

17 Vgl. auch Pampel/Tullney (2017), S. 162.

18 Siehe dazu Speicher et al. (2018) sowie den im Rahmen des Teilprojekts 4 von AT2OA verfassten Überblick von Reckling/Ferus (2019).

19 Vgl. zu dieser Problematik auch Bruch et al. (2014), S. 13 sowie Pampel/Tullney (2017), S. 163.

\section{Literatur}

Bruch, C., Fournier, J., Pampel, H. (2014): Open-Access-Publikationsfonds: Eine Handreichung, (Arbeitsgruppe Open Access der Schwerpunktinitiative „Digitale Information” der Allianz der deutschen Wissenschaftsorganisationen, Ed.). http://doi.org/10.2312/allianzoa.006

Bruch, C., Deinzer, G., Geschuhn, K., Hätscher, P., Hillenkötter, K., Kreß, U., Pampel, H., Schäffler, H., Stanek, U., Timm, A., Wagner, A. (2015): Positionen zur Schaffung eines wissenschaftsadäquaten Open-Access-Publikationsmarktes: Positionspapier der Ad-hoc-AG Open-Access-Gold im Rahmen der Schwerpunktinitiative „Digitale Information“ der Allianz der deutschen Wissenschaftsorganisationen. http://doi. org/10.2312/allianzoa.008

Capellaro, C., Kaier C. et al. (2019): Open-Access-Publikationsfonds. Einrichtung und Förderbedingungen. http://doi.org/10.5281/zenodo. 2653725

Fessler, G., Hölbling, L. (2019): Ausbau von Open Access an den österreichischen Universitäten: Budgetärer Mehrbedarf für die Jahre 20192021. http://doi.org/10.5281/zenodo.2621015 
Pampel, H., Tullney, M. (2017): 3b. Open-Access-Publikationsfonds. In: Konstanze Söllner, Bernhard Mittermaier (Eds.), Praxishandbuch Open Access, 162-172. Berlin, Boston: De Gruyter. http://doi. org/10.1515/9783110494068-019

Reckling, F., Ferus, A. (2019): Die Förderung von alternativen, nicht-kommerziellen Open-Science-Infrastrukturen \& -Services (OSIS) durch Forschungseinrichtungen in Österreich - Empfehlungen, Kriterien und Modelle. http://doi.org/10.5281/zenodo.2549452

Speicher, L., Armando, L., Bargheer, M., Eve, M., Fund, S., Leão, D., Mosterd, M., Pinter, F., Souyioultzoglou, I. (2018): OPERAS Open Access Business Models White Paper. http://doi.org/10.5281/zenodo. 1323708

TU9 German Universities of Technology e. V. (2018): TU9-Handreichung zu einheitlichen Kriterien für Open-Access-Publikationsfonds. https:// www.tu9.de/media/docs/tu9/TU9-Handreichung_einheitliche_Kriterien_OA-Publikationsfonds_12-2018.pdf\#Handreichung\%20Kriterien\%200A-Publikationsfonds 